\title{
INTER-INDUSTRY COLLABORATION: A PRACTICAL AND THEORETICAL EXAMINATION
}

\author{
Woonghee Lee, Hanyang University, Seoul, Korea
}

dx.doi.org/10.18374/RBR-19-2.4

\begin{abstract}
Most inter-firm collaboration and strategic alliance literature focus on intra-industry collaborations, neglecting inter-industry collaborations. Considering these limitations, this paper aims to illuminate the importance of inter-industry collaboration by highlighting the critical difference between intra-industry and inter-industry collaboration. Then, two different types of inter-industry collaborations - brand collaboration and innovation collaboration - will be contrasted and compared using well-known successful collaboration examples. Finally, this paper proposes a third type of inter-industry collaboration, which involves both brand and innovation collaboration, and discusses its characteristics regarding resource combination and the uncertainty that partner firms perceive.
\end{abstract}

Keywords: Inter-industry collaboration; Intra-industry collaboration; alliance 